\title{
¿La diabetes tipo 2 debería diagnosticarse y tratarse como una enfermedad cardiovascular?
}

\author{
J. M. Millaruelo Trillo, J. Sangros González, F. Remiro Serrano* \\ Medico de Familia. *Residente de 3o año de Medicina de Familia. \\ Centro de Salud Torrero La Paz. Zaragoza
}

\section{RESUMEN}

Cada vez más estudios clínicos ponen de mani fiesto que existe una asociación entre diabetes tipo 2 y enfermedad cardiovascular, y que la mayoría de los pacientes diabéticos fallecen debido a com plicaciones macrovasculares.

Estudios prospectivos recientes han demostrado que un buen control de la glucemia en ayunas y de la hemoglobina glucosilada, es capaz de prevenir la aparición de las complicaciones microvascula res en los diabéticos. Otros estudios epidemiológi cos han resaltado la importancia de la hipergluce mia post-prandial y su papel como factor de riesgo independiente de morbi-mortalidad cardiovascu lar. Los individuos con el llamado síndrome meta bólico (hipertensión arterial, dislipemia, obesidad central, insulin-resistencia e hiperinsulinismo) tie nen un riesgo elevado de padecer una enfermedad cardiovascular, sin tener una diabetes manifiesta sino, solamente, una tolerancia alterada a la glu cosa. Esto pone de manifiesto que el riesgo cardio vascular aumenta de forma paralela al incremento glucémico global (glucemia basal y post-prandial).

Por ello, las opciones terapéuticas actuales, en caminadas a controlar las alteraciones metabóli cas de la diabetes, deben tratar, también, de preve nir a largo plazo la aparición de las complicaciones macrovasculares de la enferme dad. La nateglinida, un estimulador de la secre ción de insulina de acción rápida y de corta dura-
Type 2 diabetes mellitus: should be diagnosed and treated as a cardiovascular disease?

\begin{abstract}
There are an increasing body of evidences from clinical trials that associate type 2 diabetes with cardiovascular disease, especially since the majo rity of those patients die from a cardiovascular disease and macrovascular complications.

Recent prospective studies have shown that achieving a good metabolic control by decreasing fasting plasma glucose and glycosylated hemoglo bin is possible to prevent the development of mi crovascular complications of type 2 diabetics. Ne vertheless, other epidemiological studies have stressed the importance of postprandial hypergly cemia and his role as an independent risk factor of cardiovascular morbidity and mortality. People af fected by the so-called metabolic syndrome (hyper tension, dyslipidemia, central adiposity, insulin re sistance and hyperisulinism) have a high risk to develop a cardiovascular disease, even without been affected by type 2 diabetes but only with im paired glucose tolerance. This showing that the cardiovascular risk is increasing at the same time as the entire glycemic levels (both mealtime glucose spikes and fasting plasma glucose).

The current therapeutical choices to correct the metabolic alterations of diabetes should also pre vent the appearance of long-term macrovascular complications of the disease. Nateglinide, a rapidonset, short-acting insulin secretion enhancer, which acts to effectively reduce mealtime glucose
\end{abstract}

Aceptación: 6-05-02 
ción, que actúa reduciendo eficazmente los picos post-prandiales de glucosa, puede ofrecer una al ternativa terapéutica válida para el manejo global del paciente con diabetes tipo 2.

Palabras clave: Diabetes mellitus tipo 2. Gluce mia postprandial. Enfermedad cardiovascular. Dislipemia. Aterosclerosis. Nateglinida. spikes, could offer a good therapeutical option to manage patients with type 2 diabetes.

Key words: Type 2 diabetes mellitus. Postpran dial glycemia. Cardiovascular disease. Dyslipide mia. Atherosclerosis. Nateglinide.

\section{ENFERMEDAD CARDIOVASCULAR, "EL ASESINO SILENTE”' EN PACIENTES CON DIABETES TIPO 2}

La enfermedad cardiovascular (ECV) es responsable del $75 \%$ de la mortalidad total en los pacientes con diabetes tipo $2^{1}$. Estos tienen un riesgo de dos a cuatro veces mayor de ECV que los pacientes con tolerancia normal a la glucosa y, en ellos, los sucesos cardiovasculares mortales son hasta 70 veces más frecuentes que las complicaciones microvasculares mortales ${ }^{2,3}$.

En EE.UU. la diabetes es la cuarta causa de mortalidad por orden de frecuencia. En España ocupa un lugar inferior debido, probablemente, a que no se considera como "causa fundamental" en los certificados de defunción, donde sí figuran las complicaciones cardiovasculares que genera.

La disminución, en los últimos años, de las complicaciones microvasculares, tradicionalmente asociadas con la diabetes, puede haber servido para resaltar la importancia de la ECV.

Un hecho muy preocupante es que las complicaciones macrovasculares de la diabetes tipo 2 representan un riesgo mayor en individuos más jóvenes. El riesgo relativo de fallecimiento debido a enfermedad cardiovascular (pacientes diabéticos en comparación con pacientes con tolerancia normal a la glucosa) disminuye desde un valor de 3,6 a los 2544 años, a 1,5 a los 65-74 años. Así pues, la diabetes tipo 2 aumenta la posibilidad de que las personas menores de 40 años desarrollen $\mathrm{ECV}^{4}$. Las implicaciones clínicas y económicas, de la alta incidencia de ECV en pacientes con diabetes tipo 2, son enormes y es de esperar que aumenten a medida que lo haga el número de diabéticos de tipo $2^{5}$.

\section{IMPACTO DE LOS FACTORES DE RIESGO DE ENFERMEDAD CARDIOVASCULAR EN LA DIABETES TIPO 2}

Hace casi 20 años se estableció un conjunto de factores de riesgo de enfermedad cardiovascular (FRCV): colesterol elevado en plasma, hipertensión y hábito tabáquico ${ }^{6}$. La presencia de uno o más de estos factores aumenta el riesgo global de $\mathrm{ECV}$, siendo el riesgo total mayor que la suma de cada factor de riesgo por separado.

La diabetes mellitus tipo 2 se acompaña de un riesgo de ECV mayor que el que presenta la población general con los mismos FRCV (Fig. 1) ${ }^{3}$. El control de todos los factores de riesgo clásicos sólo logra eliminar la mitad del exceso de riesgo de ECV.

Los procesos fisiológicos que explican este aumento del riesgo de ECV no se comprenden totalmente, pero se sabe que están implicados un control deficiente de la glucemia, la dislipemia, la hipertensión arterial, la hiperinsulinemia/resistencia a la insulina y la adiposidad central ${ }^{8}$.

\section{HIPERGLUCEMIA Y RIESGO CARDIOVASCULAR}

La notable disminución de las complicaciones microvasculares y la tendencia a la reducción de las complicaciones macrovasculares, después de tratamiento intensivo en pacientes con diabetes tipo 1 , ha propiciado un enorme interés por los efectos de la hiperglucemia en los pacientes con diabetes tipo 2. Muchos estudios han confirmado el papel importante de la hiperglucemia en el desarrollo de ECV, así como los efectos positivos de un control intensivo de la glucosa sobre la reducción de la enfermedad ${ }^{2,10,11}$.

A mediados de la década de 1990, cuando se realizaron estos ensayos, se pensaba que la hiperglucemia en ayunas era la causa principal de las complicaciones diabéticas y el control se basaba en la determinación de la glucosa plasmática en ayunas y la concentración de hemoglobina glucosilada $\left(\mathrm{HbA}_{1 \mathrm{C}}\right)$.

Más recientemente, se ha demostrado que la glucosa plasmática en ayunas y la $\mathrm{HbA}_{1 \mathrm{C}}$ no explican completamente el riesgo cardiovascular de los pacientes diabéticos, y que otros factores, especialmente los picos postprandiales de glucosa, aumentan el riesgo de $\mathrm{ECV}^{11,14}$. En estos estudios, la concentración de glucosa a las 2 horas de la ingesta, la hiperglucemia después de la administración 

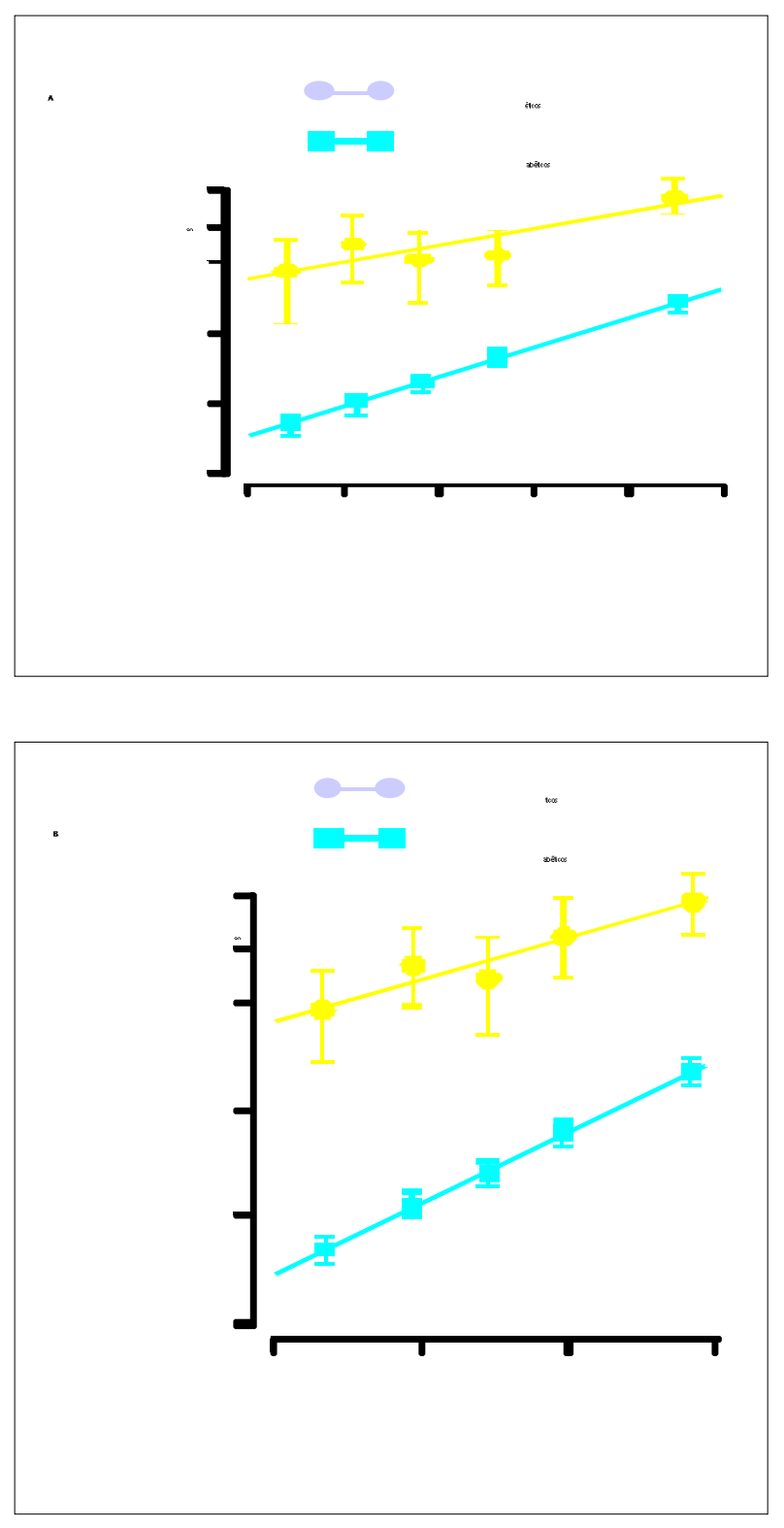

Figura 1

Riesgo de enfermedad cardiovascular en la diabetes tipo 2 , independientemente de los factores de riesgo

clásicos.

En cada gráfico, la línea superior corresponde a la diabetes y la inferior a la tolerancia normal a la glucosa. Adaptado con autorización, de Stamler J, et al 1993.

de glucosa y la intolerancia a la glucosa, se identificaron como factores de riesgo independientes de ECV, no así la glucosa plasmática en ayunas.

A medida que ha evolucionado nuestro conocimiento de la relación entre hiperglucemia y ECV, también ha aumentado la comprensión de la necesidad de reducir lo más posible el tiempo de exposición a la hiperglucemia, mediante un diagnóstico precoz y un control más estrecho del riesgo glucé- mico global (picos post-prandiales de glucosa y glucosa plasmática en ayunas). La mayoría de los casos de diabetes tipo 2 van precedidos de un periodo asintomático de intolerancia a la glucosa y, hasta hace muy poco tiempo, no estaba claro si la concentración sanguínea de glucosa representaba un mayor riesgo de ECV. Actualmente, parece que este riesgo no se limita a la intolerancia a la glucosa y diabetes tipo 2 sino que se trata de un efecto paralelo al incremento glucémico ${ }^{15,17}$.

En el Framingham Offspring Study, con el fin de explicar el exceso de riesgo cardiovascular de los pacientes con intolerancia a la glucosa no achacable a FRCV conocidos, se estudió a individuos sin enfermedad cardiovascular según tuviesen una tolerancia normal a la glucosa $(80,2 \%)$, intolerancia $(15,2 \%)$ o diabetes mellitus sin diagnosticar $(4,7 \%)^{18}$. El número de episodios de cardiopatía coronaria, mortales y totales, aumentó a medida que lo hicieron las concentraciones de glucosa, independientemente de otros FRCV conocidos ${ }^{19}$ (Fig. 2)

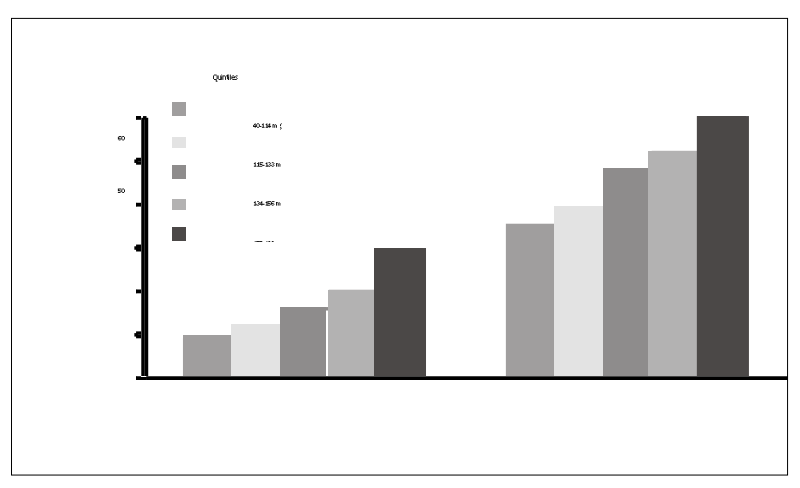

Figura 2

Relación positiva continua entre concentración de glucosa después de la comida y enfermedad coronaria (cardiopatía coronaria). Adaptado con autorización de Donahue RP, et al. Diabetes 1987; 36: 689-92.

\section{DISLIPEMIA Y RIESGO DE ENFERMEDAD CARDIOVASCULAR}

La dislipemia es un factor de riesgo muy importante de cardiopatía coronaria (CC) en pacientes con diabetes tipo 2.

En contraste con los estudios sobre la hiperglucemia, existen relativamente pocos estudios prospectivos sobre el valor predictivo de CC de los lípidos y las lipoproteínas, en pacientes con diabetes tipo 2. Aunque existe cierta controversia sobre si el aumento de los triglicéridos constituye un factor de riesgo primario, los pacientes con diabetes tipo 2 y cardiopatía coronaria tienen concentraciones más altas de triglicéridos totales y de triglicéridos-VLDL (VLDL $=$ very-low-density-lipoprotein $=$ lipoproteína de 
muy baja densidad) que los pacientes diabéticos sin cardiopatía coronaria ${ }^{20,21}$. El tratamiento de la dislipemia diabética se ha centrado históricamente en la reducción de la concentración de LDL (LDL = lowdensity-lipoprotein = lipoproteína de baja densidad) pero, en la diabetes tipo 2, la elevación de los triglicéridos puede ser mejor marcador de riesgo de CC que las cifras altas de LDL, ya que muestran mejor correlación con otros componentes del síndrome de resistencia a la insulina. El descenso de la concentración de triglicéridos en plasma puede contribuir a aumentar las concentraciones de HDL (HDL = highdensity-lipoprotein $=$ lipoproteína de alta densidad) $\mathrm{y}$, al mismo tiempo, reducir la concentración del in hibitor-1 del activador del plasminógeno, con lo que disminuye el riesgo de formación de ateromas. Además, en pacientes con diabetes tipo 2, existe una relación positiva entre concentración elevada de triglicéridos e hiperglucemia, de tal forma que el control de los picos postprandiales de glucosa se acompaña de una disminucion postprandial de triglicéridos en plasma $^{22,23}$.

\section{MECANISMO DEL AUMENTO DE RIESGO DE ENFERMEDAD CARDIOVASCULAR EN LA DIABETES TIPO 2}

La hiperglucemia contribuye a acelerar la aterogénesis y, de esta manera, al aumento de la ECV a través de diversos y complejos mecanismos, hasta ahora poco comprendidos. Cada vez hay más pruebas de que el estrés oxidativo junto con la producción de productos avanzados de la glicación, íntimamente relacionados con las anormalidades metabólicas post-prandiales, pueden tener un papel crucial en la etiología de las complicaciones vasculares $^{24}$.

Los pacientes con diabetes tipo 2 tienen concentraciones significativamente más elevadas de hidroperóxidos y, por consiguiente, una disminución del pool de antioxidantes. Este hecho no parece que esté relacionado con las anomalías del metabolismo lipídico sino con el propio síndrome de la diabetes. Parece que, en fase postprandial, la hiperglucemia combinada con la elevación de triglicéridos genera estrés oxidativo, lo cual provoca una depleción sérica y celular de antioxidantes como el alfa-tocoferol (Fig. 3) ${ }^{25}$. El estrés oxidativo puede agravar la disfunción endotelial y fomentar la aterosclerosis. Incluso una elevación temporal de la concentración de glucosa después de una comida puede tener efectos intensos y prolongados sobre el desarrollo de aterosclerosis (Fig. 4) ${ }^{26}$.

La asociación entre hiperglucemia, hipertrigliceridemia y aterogénesis se hace más compleja por los efectos de otros factores de riesgo, como la hiperinsulinemia, la resistencia a la insulina y el aumento

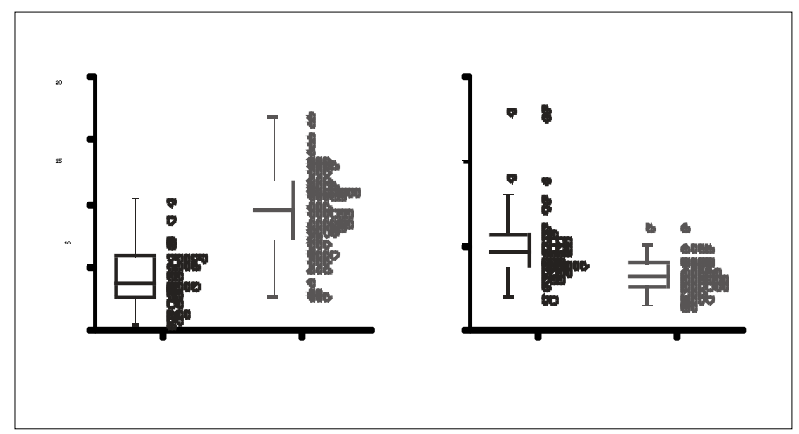

Figura 3

La diabetes tipo 2 se caracteriza por aumento del estrés oxidativo (p.e. formación del hidroperóxidos) y reducción de la concentración de antioxidantes

(p.e. alfa-tocoferol). Reproducido con autorización de Nourooz-Zadeh J, et al. Diabetología 1997; 40:

647-653.

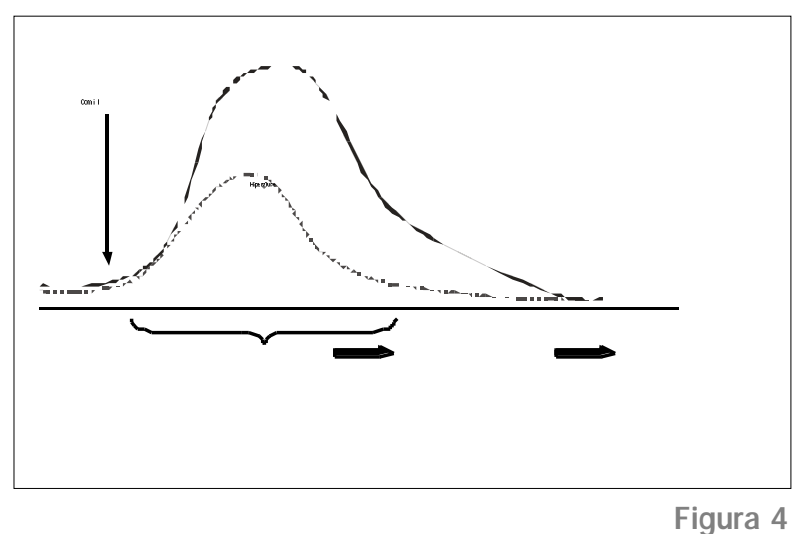

Efecto de la condición post-prandial en la pared vascu-

lar.

Adaptado con autorización de Haller $\mathrm{H}$, et al. Diab Res Clin Pract 1998; 40: S43-S49.

de la adiposidad central (más frecuente en pacientes con hiperglucemia), que pueden coexistir y aumentar el riesgo de ECV. La contribución relativa de estos factores al desarrollo de ECV en pacientes con diabetes tipo 2 es actualmente objeto de debate.

\section{ESTRATEGIAS DE TRATAMIENTO PARA REDUCIR EL RIESGO DE ENFERMEDAD CARDIOVASCULAR EN LA DIABETES TIPO 2}

El aumento del riesgo de ECV y la implicación de factores de riesgo cardiovascular modificables, proporciona una excelente oportunidad para controlarlos de un modo agresivo, usando pautas terapéuticas apropiadas.

Inicialmente, los cambios saludables del estilo de vida pueden ser suficientes para lograr una regulación satisfactoria de la glucemia, valorada 
mediante la glucosa plasmática en ayunas y la $\mathrm{HbA}_{1 \mathrm{C}}$. Sin embargo, es muy importante que los médicos no consideren que el problema está resuelto cuando la concentración plasmática de glucosa en ayunas está controlada mediante dieta y ejercicio. Deben ser conscientes del riesgo oculto de los picos post-prandiales de glucosa, su influencia en la afectación vascular y en el conjunto de alteraciones metabólicas presentes en la diabetes.

Cuando la afección progresa, la hiperglucemia persistente debida a la disminución de la secreción de insulina generalmente justifica el uso de fármacos.

La diabetes tipo 2 tiene un tratamiento muy diverso: medidas higiénico dietéticas (dieta y ejercicio), fármacos orales e insulina. La fisiopatología y la evolución de la enfermedad nos ayudarán a elegir la terapia más adecuada.

En el momento actual contamos con un importante arsenal terapéutico: sulfonilureas, biguanidas, inhibidores de las alfa-glucosidasas, glitazonas, secretagogos de acción rápida y, finalmente, la insulina.

Las sulfonilureas están indicadas en los diabéticos tipo 2 no obesos y que no se controlan con medidas higiénico dietéticas. Todas son iguales de eficaces a dosis equipotentes. Su principal efecto secundario son las hipoglucemias.

La repaglinida pertenece a la familia de las meglitinidas. Estimula a la célula beta para que libere insulina de forma más precoz que las anteriores. Se administra justo antes de las comidas. También puede ocasionar hipoglucemias.

Tanto las sulfonilureas como la repaglinida se usarán en pacientes que tengan, lógicamente, reserva pancreática.

La metformina está indicada en pacientes obesos. Tiene un efecto positivo sobre el perfil lipídico del paciente. Actúa disminuyendo la resistencia insulínica. Las alteraciones gastrointestinales, meteorismo y diarrea, son sus principales efectos secundarios.

Las sulfonilureas, la repaglinida y la metformina tienen un efecto equivalente sobre la glucemia basal (la disminuyen de 60 a $80 \mathrm{mg} / \mathrm{dl}$ ) y sobre la hemoglobina glucosilada (provocan descensos del 1,5 al $2 \%$ ).

Las glitazonas tienen un menor efecto para disminuir la glucemia basal. Se usan en combinación con metformina o sulfonilureas. Afectan negativamente al perfil lipídico de los pacientes. Sólo disminuyen del 0,5 al $1 \%$ la $\mathrm{HbA}_{1 \mathrm{C}}$.

Los inhibidores de las alfa-glucosidasas se emplean en la hiperglucemia postprandial. Actúan inhibiendo la absorción de glucosa y producen flatulencia. Logran descensos de 50 a $60 \mathrm{mg} / \mathrm{dl}$ en la glucemia postprandial y del 0,5 al $1 \%$ en la hemoglobina glucosilada.

La insulina podemos emplearla sola o en combinación con hipoglucemiantes orales. En ocasio- nes se emplea de forma transitoria: importantes descompensaciones metabólicas, accidentes vasculares, infecciones graves, etc. Otras veces, se utiliza como tratamiento definitivo: pacientes con mal control a pesar del uso de hipoglucemiantes orales a dosis máximas, diabetes LADA (latent autoimmune diabetes in adult), y otras formas de diabetes tipo 2 en las que sospechamos un déficit insulínico severo.

Antes de la introducción de la nateglinida, un estimulador de la secreción de insulina de acción rápida y corta duración, que actúa directamente sobre las células $\beta$ del páncreas, los fármacos disponibles para reducir eficazmente los picos postprandiales de glucosa eran limitados. Los inhibidores de las alfa-glucosidasas (acarbosa) se asocian con efectos secundarios gastrointestinales y un elevado porcentaje de suspensión del tratamiento. La repaglinida (derivado del ácido benzoico) tiene una acción algo más prolongada sobre las células $\beta$. Existen algunas pruebas preliminares de que la rosiglitazona (una tiazolidindiona) puede tener efecto sobre los picos postprandiales de glucosa, pero todavía no se ha confirmado su eficacia y tolerabilidad a largo plazo.

Los resultados de los ensayos clínicos con nateglinida, que actualmente está autorizada en varios países de todo el mundo (EE.UU., Japón y Suiza), indican que el fármaco es eficaz para regular los picos post-prandiales de glucosa y, posiblemente, para reducir el riesgo de $\mathrm{ECV}^{27,28}$.

El objetivo del tratamiento de la diabetes tipo 2 debería ser controlar todos los factores de riesgo modificables de ECV. Con frecuencia, el tratamiento de primera línea de estos trastornos metabólicos no tiene éxito cuando se basa en la modificación de hábitos, aumento de la actividad física y pérdida de peso. Por consiguiente, habitualmente se necesita tratamiento farmacológico.

Los pacientes con diabetes tipo 2, que presentan hiperglucemia y elevación de LDL, son tratados, a menudo, con asociación de un hipoglucemiante y una estatina ${ }^{29}$. Sin embargo, las estatinas son sólo moderadamente eficaces para reducir la concentración de triglicéridos, por lo que la reducción de la hipertrigliceridemia precisa, en ocasiones, la asociación de un fibrato. La asociación de estatinas y ácido nicotínico es eficaz para modificar las dislipidemias diabéticas, pero puede empeorar significativamente la hiperglucemia ${ }^{30}$.

El desafío es hallar una pauta terapéutica que controle, con eficacia, todos los factores de riesgo que predisponen a los pacientes con diabetes tipo 2 a sufrir ECV.

La asociación de antidiabéticos orales con mecanismo de acción complementario como, por ejemplo, nateglinida (estimulador de la secreción de insulina que controla la hiperglucemia post-prandial) 
y metformina (sensibilizador de la insulina que disminuye los triglicéridos en plasma), constituye una estrategia eficaz para reducir el riesgo de ECV en pacientes con diabetes tipo 2 .

\section{CONCLUSIONES}

Durante los últimos años, la reducción de las complicaciones microvasculares asociadas con la diabetes tipo 2 , ha servido para destacar la magnitud de las complicaciones macrovasculares de esta afección, cada vez más frecuente. El $80 \%$ de la mortalidad relacionada con la diabetes es consecuencia de sucesos cardiovasculares. El tratamiento de la diabetes tipo 2 está cambiando espectacularmente, al conocerse que la enfermedad cardiovascular aparece antes que la elevación de las cifras de glucemia basal y que las complicaciones microvasculares. El control de la glucemia post-prandial y de los factores de riesgo cardiovascular deben ser objetivos irrenunciables para estos pacientes.

Según esto, la diabetes tipo 2 debería actualmente diagnosticarse y tratarse precozmente como una enfermedad cardiovascular.

\section{CORRESPONDENCIA:}

José Manuel Millaruelo Trillo

Centro de Salud Torrero La Paz

C/ Soleiman 11

50007 Zaragoza

e-mail: : jmmillaruelo@able.es

Fax: 976-254006. Tel.: 976-254266.

\section{Bibliografía}

1. Geiss LS, Herman WH, Smith PJ. Mortality in non-insulindependent diabetes. En: Harris M, ed. Diabetes in America, Second Edition Bethesda: National Institutes of Health, 1995; p. 429-56.

2. UKPDS 33 (1998). Intensive blood glucose control with sulphonylureas or insulin compared with conventional treatment and risk of complications in patients with type 2 diabetes. UK Prospective Diabetes Study (UKPDS) Group. Lancet 1998; 352: 837-53.

3. Stamler J, Vaccaro O, Neaton J, Wentworth D. For the Multiple Risk Factor Intervention Trial Research Group. Diabetes, other risk factors and 12-yr cardiovascular mortality for men screening in the Multiple Risk factor Intervention Trial. Diabetes Care 1993; 16: 434-44.

4. Claudi T, Midthjell K, Holmen J, Fougner K, Kruger O, Wiseth R. Cardiovascular disease and risk factors in persons with type 2 diabetes diagnosed in a large population screening: the Nord-Trondelag Diabetes Study, Norway. J Intern Med 2000; 248: 492-500.

5. Amos AF, McCarty DJ, Zimmet P. The rising global burden of diabetes and its complications: estimates and projections to the year 2010. Diabet Med 1997; 14: S1-85.

6. Multiple Risk Factor Intervention Trial Research Group: Multiple Risk Factor Intervention trial risk factor changes and mortality results. JAMA 1982; 248: 1465-76.

7. Eastman RC, Keen H. The impact of cardiovascular disease on people with diabetes: treatment and prevention. Lancet 1997; 350 (Supl. 1): 29S-32S.

8. Ceriello A. The post-prandial state and cardiovascular disease: relevance to diabetes mellitus. Diabetes Metab Res Rev 2000; 16: 125-32.

9. The DCCT Research Group. The relationship of glycemic exposure (HbA1C) to the risk of development and progression of retinopathy in the Diabetes Control and Complications Trial. Diabetes 1995; 44: 968-83.

10. Hanefeld M, Fischer S, Julius U, Schulze J, Schwanebeck $\mathrm{U}$, Schmechel $\mathrm{H}$, et al. The DIS Group. Risk factors for myocardial infarction and death in newly detected NIDDM: the Diabetes Intervention Study, 11-year follow-up. Diabetologia 1996; 39: 1577-83.

11. Shaw JE, Hodge AM, de Courten M, Chitson P, Zimmet PZ. Isolated post-challenge hyperglycemia confirmed as a risk factor for mortality. Diabetologia 1999; 42: 1050-4.

12. Barrett-Connor E, Ferrara A. Isolated post-challenges hyperglycemia and the risk of fatal cardiovascular disease in older women and men. The Rancho Bernardo Study. Diabetes Care 1998; 21: 1236-9.

13. The DECODE study group on behalf of the European Diabetes Epidemiology Group Glucose tolerance and mortality: comparison of WHO and American Diabetes Association diagnostic criteria. Lancet 1999; 354: 617-21.

14. Tominaga M, Eguchi H, Manaka H, Igarashi K, Kato T, Sekikawa A. Impaired glucose tolerance is a risk factor for cardiovascular disease, but not impaired fasting glucose. The Funagata Diabetes Study. Diabetes Care 1999; 22: 920-4.

15. Coutinho M, Gerstein H, Wang Y, Yusuf S. The relationship between glucose and incident cardiovascular events. A metaregression analysis of published data from 20 studies of 95,783 individuals followed for 12.4 years. Diabetes Care 1999; 22: 233-40.

16. Khaw KT, Wareham N, Luben R, Bingham S, Oakes S, Welch A, et al. Glycated haemoglobin, diabetes and mortality in men in Norfolk cohort of European prospective investigation of cancer and nutrition (EPIC-Norfolk). BMJ 2001; 322: 1-6.

17. Barrett-Connor E. "Normal" blood glucose and coronary risk. BMJ 2001; 322: 5-6.

18. Meigs JB, Singer DE, Nathan DM. Metabolic abnormalities with glucose intolerance extend across the spectrum of prevalent glucose tolerance in 3297 Framingham Offspring Study patients. Diabetes 1995; 44 (Supl. 1): 5A.

19. Donahue RP, Abbott RD, Reed DM, Yano K. Postchallenge glucose concentration and coronary heart disease in men of Japanese ancestry. Diabetes 1987; 36: 689-92.

20. Laakso M, Lehto S, Penttila I, Pyorala K. Lipids and lipoproteins predicting coronary heart disease mortality and 
morbidity in patients with non-insulin-dependent diabetes. Circulation 1993; 88: 1421-30.

21. Patsch JR, Miesenbock G, Hopferweiser T, Muhlberger V, Knapp E, Dunn JK, et al. Relation of triglyceride metabolism and coronary artery disease. Arteriosclerosis and Thrombosis 1992; 12 (11): 1336-45.

22. Howard BV. Lipoprotein metabolism in diabetes mellitus. J Lipid Res 1987; 28: 613-28.

23. Hanefeld M, Fischer S, Schulze J, Splenger M, Wargenau $\mathrm{M}$, Schollberg K, et al. Therapeutic potentials of acarbose as first-line drug in NIDDM insufficiently treated with diet alone. Diabetes Care 1991; 14: 732-7.

24. Giugliano D, Ceriello A, Paolisso G. Oxidative stress and diabetic vascular complications. Diabetes Care 1996; 19: 257-67.

25. Nourooz-Zadeh J, Rahimi A, Tajaddini-Sarmadi J, Tritschler H, Rosen P, Halliwell B, et al. Relationships between plasma measures of oxidative stress and metabolic control in NIDDM. Diabetologia 1997; 40: 647-53.
26. Haller $\mathrm{H}$. The clinical importance of postprandial glucose. Diab Res Clin Pract 1998; 40 (Supl.): 43S-49S.

27. Hanefeld M, Bouter KP, Dickinson S, Guitard C. Rapid and short-acting mealtime insulin secretion with nateglinide controls both prandial and mean glycemia. Diabetes Care. 2000 Feb; 23 (2): 202-7.

28. Horton E, Clinkingbeard C, Gatlin M, Foley J, Mallows S, Shen S. Nateglinide alone and in combination with metformin improves glycemic control by reducing mealtime glucose spikes in type 2 diabetes. Diabetes Care 2000; 23 : 1660-5.

29. Kreisberg RA. Diabetic dyslipidemia. Am J Cardiol 1998; 82 (12A): 67U-73U.

30. Lithell H, Vessby B, Hellsing K. Changes in glucose tolerance and plasma insulin during lipid lowering treatment with diet, clofibrate and niceritrol. Atherosclerosis 1982; 43: $177-84$. 